\title{
CHARMM force field parameterization protocol for self- assembling peptide amphiphiles: The Fmoc moiety
}

Received 00th January 20xx Accepted 00th January $20 x x$ DOI: $10.1039 / x 0 x \times 00000 x$ www.rsc.org/

\begin{abstract}
I. Ramos Sasselli, ${ }^{a}$ R. V. Ulijn ${ }^{a, b}$ and T. Tuttle ${ }^{a, *}$
Aromatic peptide amphiphiles are known to self-assemble into nanostructures but the molecular level structure and the mechanism of formation of these nanostructures is not yet understood in detail. Molecular Dynamic simulations using the CHARMM force field have been applied to a wide variety of peptide-based systems to obtain molecular level details of processes that are inaccessible with experimental techniques. However, this force field does not include parameters for the aromatic moieties which dictate the self-assembly of these systems. The standard CHARMM force field parameterization protocol uses hydrophilic interactions for the non-bonding parameters evaluation. However, to effectively reproduce the self-assembling behaviour of these molecules, the balance between the hydrophillic and hydrophobic nature of the molecule is essential. In this work, a modified parameterization protocol for the CHARMM force field for these aromatic moieties is presented. This protocol is applied for the specific case of the Fmoc moiety. The resulting set of parameters satisfies the conformational and interactions analysis and is able to reproduce experimental results such as the Fmoc-S-OMe water/octanol partition free energy and the self-assembly of $\mathrm{Fmoc}-\mathrm{S}-\mathrm{OH}$ and $\mathrm{Fmoc}-\mathrm{Y}-\mathrm{OH}$ into spherical micelles and fibres, respectively, while also providing detailed information on the mechanism of these processes. The effectiveness of the parameters for the Fmoc moiety validates the protocol as a robust approach to paramterise this class of compounds.
\end{abstract}

\section{Introduction}

Peptides are known to self-assemble into nanostructures with very interesting applications in nanotechnology and biomedicine..$^{1-3}$ The supramolecular functionality of peptidebased nanomaterials is due not only to the functional groups present in the molecules but also due to the supramolecular structures formed. ${ }^{4}$ Self-assembly is a spontaneous process driven by intermolecular interactions such as electrostatic interactions, hydrogen bonding, $\pi$-stacking and the hydrophobic effect. The amino acid sequence of the peptide building blocks affects the ability of the peptide to form these interactions and, therefore, different amino acids sequences can lead to different structure types such as fibres, ribbons, tubes, and sheets. ${ }^{5-8}$ Understanding how the amino acid sequence affects the final structure is of particular interest for elucidating the "design rules" for peptide based nanomaterials.

Computational methods have been employed with

\footnotetext{
a. Pure and Applied Chemistry Department, WestCHEM. University of Strathclyde. 295 Cathedral Street, Glasgow, G1 1XL, UK.

${ }^{b .}$ Advanced Science Research Center (ASRC) and Hunter College, City University of New York, 85 St Nicholas Terrace, New York, New York 10031, USA.

*E-mail: tell.tuttle@strath.ac.uk

Electronic Supplementary Information (ESI) available: topology and parameters for the Fmoc moiety in the NAMD format, further details of the MD-FEP solvation free energy results, the Fmoc-S-OH experimental particle analysis and the details on the experimental determination of the Fmoc-S-OMe partition coefficient. See DOI: $10.1039 / x 0 x x 00000 x$
}

significant success to reveal both molecular level detail and the mechanism of formation for a range of peptide-based nanostructures. Molecular Dynamics (MD) simulations have been applied to increase the understanding of different features of peptide-based nanomaterials at the molecular level. ${ }^{9,} 10$ In particular, coarse grain (CG) simulations have been increasingly utilized to illustrate the aggregation of peptides and peptide amphiphiles (PA). ${ }^{11}$ CG simulations have also provided insights into different self-assembling mechanism by studying the free energy profiles of these processes, ${ }^{12}$ and a systematic CG screening approach was applied to all possible di- and tripeptides and has successfully described literature examples of self-assembled peptides and predicted the selfassembling tendency of other peptides that could subsequently be verified by experimentation. ${ }^{13,14}$

Atomistic MD simulations have also been used in the area of self-assembling materials, although primarily this has been to study the preferred conformations of the peptides or PA in the assembled state. ${ }^{15-17}$ Atomistic simulations are limited to smaller sizes than $\mathrm{CG}$ and require substantially more computation time but they have the advantage of atomistic resolution, which is required for the study of the specific noncovalent interactions involved in the self-assembly process. Free energy profile studies using atomistic MD simulations have also been successfully applied for the study of selfassembling mechanism of aliphatic PAs. ${ }^{18,19}$

One important class of PA is the aromatic peptide amphiphiles, which are formed by a peptide capped at the $\mathrm{N}$ - 
terminus with an aromatic group. ${ }^{20-22}$ The formation of extra $\pi$-stacking interactions by the aromatic group facilitates the self-assembly in these systems and allows shorter peptides to form nanostructures. ${ }^{23}$ Several di- and tripeptides bound to the fluorenyl-9-methoxycarbonyl (Fmoc) group have been found to form nanostructures which are strongly dependent of the amino acid sequence (e.g., Fmoc-TF-OMe self-assembles into fibres while Fmoc-SF-OMe forms sheets). ${ }^{4,}$, 7, 24-26 There are even examples of single amino acids protected with the Fmoc moiety which are able to form nanostructures. ${ }^{27-29}$ As such, this class of compounds represent a highly tuneable set of PAs that may be used for a wide range of applications. Remarkably, it is not only the fluorenyl group but also the specific geometry of the methoxycarbonyl linker which aids in the self-assembly process as was recently demonstrated by varying the linker between fluorenyl and peptide for FmocYL. ${ }^{30}$

As in the case of other PAs, the structures formed by Fmocpeptides are not fully understood and often discovered by chance rather than rational molecular design. MD methods have been already applied to these systems in order to gain understanding on the preferred conformations of these molecules in the self-assembled state and on the importance of the different interactions in the self-assembling mechanism. ${ }^{15}$, 31-33 However, the implementation of simulations for these systems has an added difficulty which is that the force fields used for the simulations do not include parameters for the Fmoc moiety. Therefore, the first step for the use of MD methods in Fmoc-peptides self-assembly is the parameterization of this aromatic moiety. As the quality of the results obtained in subsequent simulations - particularly in the case of unbiased, long timescale simulations - will be dependent on the quality of the parameters used, an accurate and consistent parameterization of the Fmoc moiety is critical.

Force field parameters are typically obtained either from experimental results or quantum mechanical data (QM). ${ }^{34-37}$ The molecules used to derive these parameters and the way they are obtained and optimized are characteristic of a force field and limit its applications. The CHARMM force field was chosen to simulate the self-assembling peptide based systems as it is parameterized and well validated for proteins and peptides. ${ }^{34,38}$ The CHARMM force field includes parameters for many organic molecules (including fluorene and other aromatic groups), lipids, nucleic acids, and some carbohydrates. ${ }^{38-40}$ It is common when parameterizing a new molecule in a force field which includes parameters for such a wide range of molecules to obtain the bonded parameters from similar segments of molecules already parameterized for that force field. ${ }^{31,41,42}$ However, the non-bonded parameters, electrostatic and van der Waals, need to be optimized. As these parameters may have an influence in the torsional potentials, the torsional parameters also need to be optimized.

The CHARMM parameterization protocol evaluates the interactions of the hydrophilic parts of a given molecule with water. $^{34,39,40}$ Nevertheless, to reproduce the self-assembling behaviour of aromatic peptide amphiphiles a balance between the hydrophilic and the hydrophobic nature of the group is essential. Therefore, in this study we present a modified protocol to parameterize aromatic moieties, within the CHARMM force field, that are used to form aromatic peptide amphiphiles. The protocol is presented for the Fmoc moiety due to the high prevalence of Fmoc-peptide amphiphiles as self-assembling molecules. ${ }^{29}$

This study is presented starting with the parameterization of the Fmoc moiety, which includes: the derivation of the bonded parameters from parameterized molecules with similar segments; the charges and van der Waals terms optimization; and the torsional terms optimization. The second part of the study is focused in the validation of the parameterization: firstly comparing the partition coefficient of Fmoc-S-OMe obtained computationally with the partition coefficient measured experimentally; and secondly by using the parameters to study self-assembling systems whose final structures are well known.

\section{Parameterization of Fmoc}

The parameterization was made for the CHARMM force field, which includes parameters for amino acids as well as a wide range of other organic molecules. To parameterize the Fmoc moiety some of the parameters where extrapolated from similar molecules or molecules which present the same chemical groups of the CHARMM parameters library.

The parameters for 9-fluorenylmethyloxycarbonyl or the Fmoc moiety were obtained as follows. For the fluorenyl group the parameters were taken from the fluorene molecule (Figure 1, Red); for the $-\mathrm{CH}_{2}-$ which links the aromatic part to the group to the oxygen, the parameters were taken from ethanol (Figure 1, Green); the parameters for the following oxygen (O-) were obtained from standard ester groups (Figure 1, Blue) and parameters from general amides were used for the carbonyl group ( $\mathrm{C}=\mathrm{O}$ ) (Figure 1, Orange).

The CHARMM force field presents the following expression for the energy:

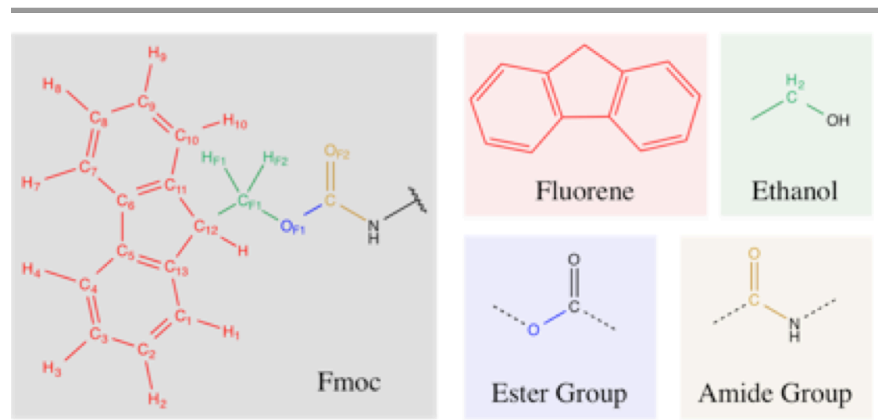

Figure 1. Atoms names for the Fmoc moiety (left) and the segments used to obtain some of its parameters (right). 


$$
\begin{aligned}
& E_{\text {ele }}(r)=\sum_{\text {bonds }} k_{b}\left(r-r_{0, b}\right)^{2}+\sum_{U B} k_{U B}\left(S-S_{0, U B}\right)^{2} \\
&+\sum_{\text {angle }} k_{a}\left(\theta-\theta_{0, a}\right)^{2} \\
&+\sum_{\text {dihedrals }} k_{d, n}\left(1+\cos \left(n \chi-\delta_{d, n}\right)\right) \\
&+\sum_{\text {impropers }} k_{i}\left(\psi-\psi_{0, i}\right)^{2} \\
&+\sum_{\text {vdw }} \varepsilon_{i j}\left[\left(\frac{R_{\text {min }, i j}}{r_{i j}}\right)^{12}-2\left(\frac{R_{\text {min }, i j}}{r_{i j}}\right)^{6}\right] \\
&+\sum_{\text {electrostatic }} \frac{q_{i} q_{j}}{4 \pi \varepsilon_{0} r_{i j}}
\end{aligned}
$$

This equation has seven different terms. The force constants $\left(k_{b}, k_{U B}, k_{a}, k_{i}\right)$ and reference values $\left(r_{0, b}, S_{0, \cup B}, \theta_{0, a}\right.$, $\left.\psi_{0, i}\right)$ for the bonds stretching, Urey-Bradley terms, angles bending and improper dihedrals were directly transferred from the above-mentioned groups in the CHARMM library. For the Lennard-Jones term, although other parameters $\left(\varepsilon_{i j}, R_{\text {min,ij }}\right)$ were tested, they did not improve the results obtained from those transferred from the same groups of the CHARMM library, hence, the transferred parameters from the mentioned segments were also used for the Lennard-Jones term. The dihedral terms $\left(k_{d, n}, n, \delta_{d, n}\right)$ for the fluorenyl were also taken from the fluorene molecule. Therefore the parameterization effort was focused on the dihedral terms of the linker between the aromatic group and the peptide and on the charges $\left(q_{i / j}\right)$, which cannot be directly transferred from the segments.

QM and MM binding energies: Fmoc - Water. To calculate the binding energies of Fmoc with water the molecule Fmoc- $\mathrm{NH}_{2}$ (Figure 2) was used. This molecule was optimized at the QM level of theory. The parameterization protocol for CHARMM uses the Hartree-Fock method with the 6-31G* basis set for the binding energies calculations. ${ }^{39}$ However, this current work includes the calculation of binding energies between water and the aromatic group, where dispersion forces may play an important role. As Hartree-Fock is unable to describe dispersion, the QM calculations were carried out using the DFT functional $B 97-D,{ }^{43}$ with the basis set def2-SVP, ${ }^{44}$ in Turbomole. ${ }^{45,46}$ The MM binding energies were calculated using the CHARMM force field with the Fmoc parameters in the NAMD programme. ${ }^{47}$

The optimized geometry of Fmoc- $\mathrm{NH}_{2}$ was fixed for all the

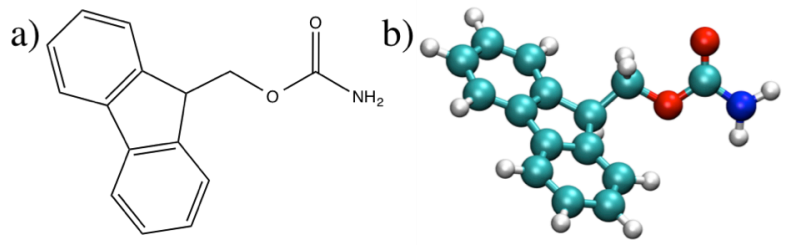

Figure 2. Fmoc- $\mathrm{NH}_{2}$ model molecule in $2 \mathrm{D}$ (a) and $3 \mathrm{D}$ (b) representations.
Fmoc - Water QM binding energies calculation. The geometry of water was also fixed in these calculations with the TIP3P geometry. ${ }^{48}$ Therefore, the binding energies (BEs) for the Fmoc - Water systems are purely interaction energies. The different Fmoc - Water systems were built in Avogadro. ${ }^{49}$ The QM optimized structures were used for the calculation of the MM binding energies. The Fmoc - Water binding energies $\left(\mathrm{BE}_{\mathrm{w}}\right)$ are calculated as the energy difference between each Fmoc Water system $\left(E_{\text {water } \cdots \mathrm{Fmoc}-\mathrm{NH}_{2}}\right)$ and the sum of the internal energies of $\mathrm{Fmoc}-\mathrm{NH}_{2} \quad\left(E_{\mathrm{Fmoc}-\mathrm{NH}_{2}}\right)$ and water ( $\left.E_{\text {water }}\right)$ (Equation 2).

$$
B E_{w}=E_{\text {water } \cdots F m o c-N H_{2}}-\left(E_{\text {water }}+E_{F m o c-N H_{2}}\right)
$$

The CHARMM parameterization protocol uses the QM reference binding energies $\left(B E_{Q M}\right)$ to optimize hydrogen bonds with water. ${ }^{34,39,40}$ However, in this work the binding energies of water with hydrophobic parts, not able to form hydrogen bonds, are also studied. This was done in order to include an additional reference to account for the hydrophilicity/hydrophobicity of the different parts of the moiety. The water - water binding energy $\left(B E_{w-w}\right)$ calculated from two water molecules with TIP3P geometry, is used as a reference point to determine the relative strength of the interactions. All $B E_{w}$ lower than this reference are considered hydrophilic, and those higher than the reference, hydrophobic. Therefore, the Final Charges Set (FCS) has to satisfy both conditions: the hydrophobic/hydrophilic behaviour of the different parts of the moiety $\left(B E_{w-w}\right)$ and the relative intensities of the interactions $\left(B E_{Q M}\right)$.

The optimized structures for the Fmoc - Water are shown in Figure 3. Structures 1 to 4 involve interactions of the water molecule with the hydrophilic parts of the Fmoc moiety, while in the structures 5 to 9 the water molecule interacts with the

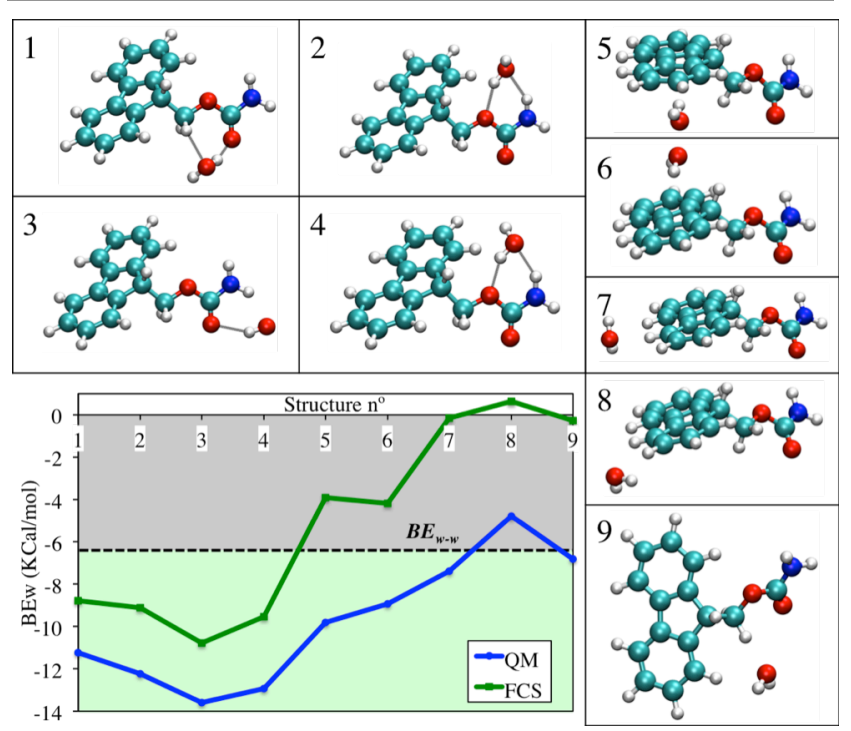

Figure 3. Optimized geometries of the Fmoc - Water systems and $B E_{w}$ graph for $F C S$ (bottom left). The graph includes two references: the dashed black line $\left(B E_{w-w}\right)$ separates the hydrophobic (grey) and the hydrophilic (lime) regions; and the $B E_{Q M}$ (blue line). 
hydrophobic parts of the moiety. Therefore the $B E_{w, 1-4}$ are expected to be lower than the $B E_{w-w}$ (lime area of the graph, Figure 3), while the $B E_{w, 5-9}$ must be higher than this reference value (grey area of the graph, Figure 3 ).

The FCS successfully reproduces the hydrophilic/hydrophobic behaviour of each part of the Fmoc moiety (Figure 3). Furthermore, the FCS reproduces also the shape of the $B E_{Q M}$ and therefore the relative intensities of the interactions are in good agreement with the QM reference. The interactions 7 to 9 , which involve the interaction of the water molecule with the edges of the aromatic group, are very weak or, even, repulsive. As this was found to happen for all charges sets trialled, it was assumed to be a limitation of the force field to reproduce this kind of interaction. Overall, the averaged absolute error for the Fmoc - Water binding energies is $4.6 \mathrm{Kcal} / \mathrm{mol}$, which is within the typical error limit of force fields.

QM and MM binding energies: Fmoc - Fmoc systems. Once the FCS was obtained, it was tested against unique interactions that the $\mathrm{Fmoc}$ parameters should reproduce to reliably describe the self-assembling behaviour, namely, the Fmoc - Fmoc interactions. Various configurations of two Fmoc$\mathrm{NH}_{2}$ molecules were built in Avogadro. The systems were optimized in Turbomole using the DFT functional B97-D with the def2-SVP basis set. The optimized geometries were used to calculate the MM energy using the CHARMM force field with the Fmoc parameters in the NAMD program. The binding energies $\left(\mathrm{BE}_{\text {dimer }}\right)$ are defined as the difference in energies between the system with two Fmoc molecules interacting

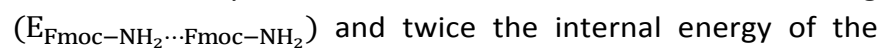
Fmoc- $\mathrm{NH}_{2}$ molecule $\left(\mathrm{E}_{\mathrm{Fmoc}-\mathrm{NH}_{2}}\right.$ ) (Equation 3). For the Fmoc Fmoc systems the $\mathrm{BE}_{\text {dimer }}$ are not purely interactions energies but they include the internal energy change.

$B E_{\text {dimer }}=E_{\mathrm{Fmoc}-\mathrm{NH}_{2} \cdots \mathrm{Fmoc}-\mathrm{NH}_{2}}-\left(2 \cdot E_{\mathrm{Fmoc}-\mathrm{NH}_{2}}\right)$

The MM binding energies calculated using the $F C S$ ( $\left.B E_{F m o c}\right)$ for these interactions are compared with $Q M$ calculated reference binding energies $\left(B E_{Q M}\right)$. The final geometries and $\mathrm{BE}$ are presented in Figure 4. Seven different types of $\pi$-stacking interactions are studied. Six of the $B E$ values are well reproduced as the $B E_{F m o c}$ calculated with the $F C S$ are within 4.5

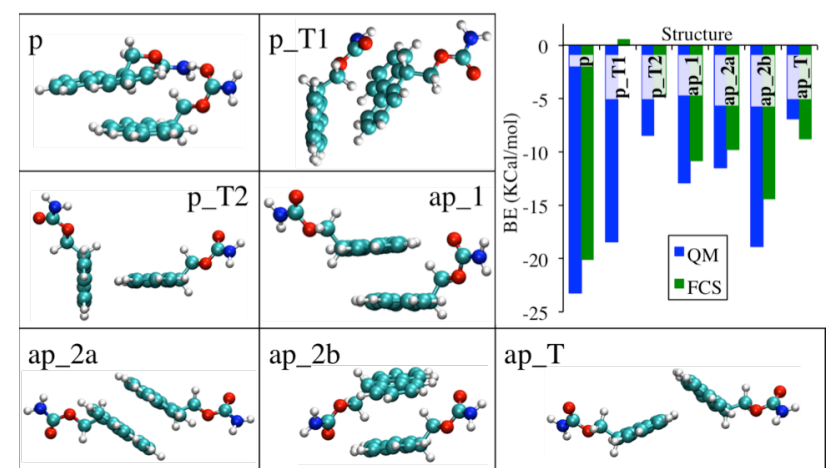

Figure 4. Optimized geometries of the $F m o c-F m o c$ systems and $B E_{\text {dimer }}$ graph for the FCS and the QM reference (top right).
$\mathrm{Kcal} / \mathrm{mol}$ from the $B E_{Q M}$. The only interaction that cannot be reproduced corresponds to the geometry $p_{-} T 1$ (Figure 4$)$ ). This geometry involves the interactions of the aromatic group of one Fmoc- $\mathrm{NH}_{2}$ with the hydrophilic part of the other molecule. As in the case of the Fmoc - Water systems 7 to 9, this is accepted as a limitation of the force field to reproduce some types of interactions. Nonetheless, the ability to successfully reproduce the six $\pi$-stacking $F m o c-F m o c$ interactions is encouraging and suggests the correct balance between the hydrophobic and hydrophilic nature of the moiety has been achieved. Furthermore, for the $\mathrm{Fmoc}-\mathrm{Fmoc}$ binding energies, the averaged absolute error is $4.6 \mathrm{Kcal} / \mathrm{mol}$, which is consistent with the error of the Fmoc - Water interactions and hence, it is also within the general accuracy of force fields.

Torsion angle parameterization. Standard amide and ester dihedral terms were used to build the dihedrals of the linker of the Fmoc. The validity of these terms was evaluated with the FCS and compared with reference QM values in order to accurately reproduce the flexibility of the Fmoc moiety. The dihedral angles under study were $\mathrm{C}_{13}-\mathrm{C}_{12}-\mathrm{C}_{\mathrm{F} 1}-\mathrm{O}_{\mathrm{F} 1}(\mathrm{~T} 1), \mathrm{C}_{12}$ $-\mathrm{C}_{\mathrm{F} 1}-\mathrm{O}_{\mathrm{F} 1}-\mathrm{C}(\mathrm{T} 2)$ and $\mathrm{C}_{\mathrm{F} 1}-\mathrm{O}_{\mathrm{F} 1}-\mathrm{C}-\mathrm{O}_{\mathrm{F} 2}(\mathrm{~T} 3)$, see Figure 1 for atom names. Torsional potential profiles were calculated in Gaussian $09^{50}$ with the B3LYP ${ }^{51,52}$ functional and the 6$31 \mathrm{G}(\mathrm{d}, \mathrm{p})$ basis set. ${ }^{53,54}$ The profiles were obtained with rigid scans, calculating the single point energies of structures generated via 10 o of rotation around each dihedral. The MM torsional potential profiles were obtained using the same geometries and calculating the energy for the CHARMM force filed with the Fmoc parameters using the NAMD program.

As well as the static $M M$ energies, the $Q M$ torsion potentials were compared with the distribution of the three dihedrals during $10 \mathrm{~ns} \mathrm{MD}$ simulations with a single molecule of Fmoc- $\mathrm{NH}_{2}$ (System 1); and with a single molecule of Fmoc-S-

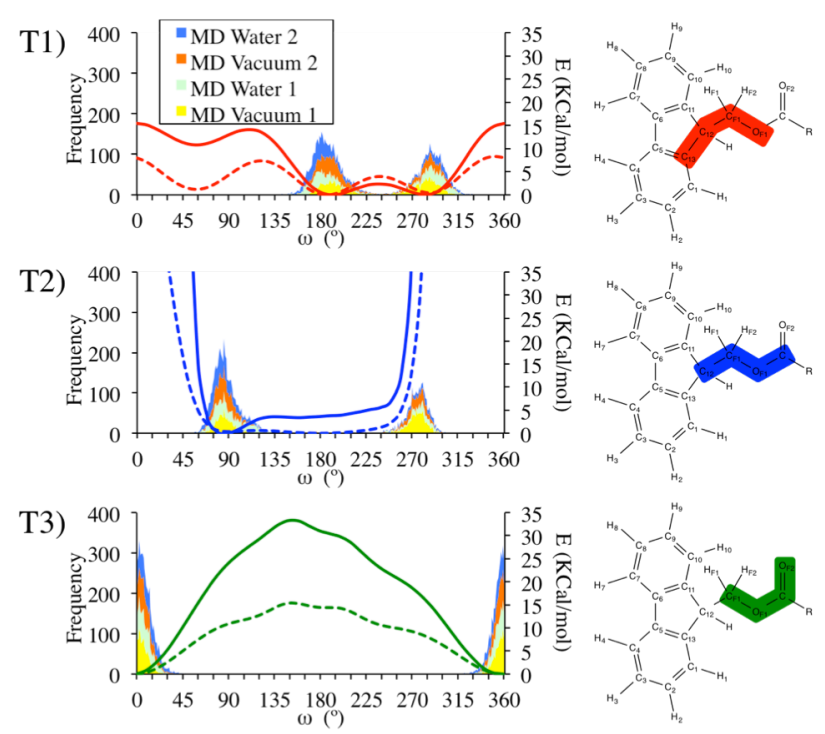

Figure 5. Torsion potentials calculated in MM (Straight lines) and QM (Dashed lines) (Right vertical axis), and torsional angles distribution in MD simulations (Stacked histograms, left vertical axis). The colour-code for the MM and QM results correspond to the images on the right and the colour-code for the histograms is on the legend of the top graph. 
$\mathrm{OH}$ (System 2). These simulations reveal the influence of incorporating the parameters of the Fmoc moiety to the parameters of the amino acids in CHARMM. Both systems were simulated in vacuum and in water (TIP3P model). The systems were built in VMD and were minimized with the steep descent technique to avoid high-energy contacts and equilibrated at $298 \mathrm{~K}$ before the simulations. The $10 \mathrm{~ns}$ simulations were carried out within the NVT ensemble and a $1.0 \mathrm{fs}$ integration time step was applied. Results are shown in Figure 5.

The MM potentials successfully reproduce the shape of the QM references (Figure 5) although the energy barriers are typically higher relative to the QM gas phase results. The distribution of dihedrals in the simulations maps well on to the QM potentials, with the majority of the torsional space explored around the regions where the potential energy is at a minimum. It can be seen that for the dihedral $T 2$ both, the MM and the QM barriers are much higher than for the other two dihedrals. This is caused by the proximity of the oxygen in the carbonyl group with the fluorenyl group during the rotation around $T 2$ for the region between 270 o and 45o degrees due to the use of a rigid scan, which cannot relax in each point of the rotation. This also causes a displacement for $T 2$ in the simulations, which shows a peak between $255^{\circ}$ and 300 o that is displaced to the right in reference to the energy potentials. This shift is caused by the difference in mobility of the systems used to calculate the different parameters: the histograms are calculated from simulations where the whole molecule can move while the $\mathrm{QM}$ and $\mathrm{MM}$ plots are calculated by rotating the dihedrals in fixed molecules. Given the agreement between the $\mathrm{QM}$ and $\mathrm{MM}$ torsional profiles and the distribution of the torsional angles from the MD simulations, the flexibility in the carbamate region of the Fmoc moiety is considered to be well reproduced.

\section{Validation via the Partition Coefficient}

As an initial validation of the parameterization carried out for the Fmoc moiety, a physical parameter, which can be directly compared with the experimental observable, is calculated. Due the amphiphilic nature of the molecules for which the Fmoc moiety is parameterized, Fmoc-peptides, and the importance of reproducing this for the self-assembly simulations, the partition coefficient between octanol and water was chosen for this purpose.

Partition coefficient determination. The molecule chosen for this validation was Fmoc-S-OMe. This serine is capped at the $\mathrm{N}$-terminus with the $\mathrm{Fmoc}$ moiety and at the $\mathrm{C}$-terminus as a methyl ester. Neither of these groups nor the serine side chain contain acidic hydrogen and therefore it is not necessary to take into account any ionization effects, which simplifies the comparison of experimental and computational results. The serine side chain was chosen due to its hydrophilicity, which facilitates the experimental determination of the log $P$.

The experimental partition coefficient is determined by the shake flask methodology ${ }^{55-57}$ as an average of 9 samples. The details of the experiment are included in the ESI. The partition

free energy $\left(\Delta G_{\text {ow }}\right)$ is related to the partition coefficient $\left(K_{\text {ow }}\right)$ using Equation 4:

$$
\Delta G_{\text {ow }}=2.303 R T \log K_{o w}
$$

The theoretical partition free energy can be calculated from the solvation free energy data of a given molecule in water $\left(\Delta G_{w}\right)$ and in octanol $\left(\Delta G_{o c}\right)$ as shown in Equation 5 :

$$
\Delta G_{o w}=\Delta G_{o c}-\Delta G_{w}
$$

The theoretical solvation free energies in water $\left(\Delta \mathrm{G}_{\mathrm{w}}\right)$ and in octanol $\left(\Delta \mathrm{G}_{\mathrm{oc}}\right)$ were calculated using alchemical methods, specifically free energy perturbation (FEP). ${ }^{58,59}$

The two systems were built in $\mathrm{VMD},{ }^{60}$ both include an Fmoc-S-OMe molecule which is surrounded by TIP3P water ${ }^{48}$ for $\Delta G_{w}$ (Figure 6a) and by octanol for $\Delta G_{\text {oc }}$ (Figure 6b). The systems were built to be $60 \times 60 \times 60 \AA$ of each solvent with the Fmoc-S-OMe placed in the centre of each phase. All MD calculations were carried out using the NAMD program and the CHARMM force field including the Fmoc parameterization presented in this paper. The systems were minimized to avoid bad contacts and then heated up and equilibrated at $298 \mathrm{~K}$ and $1 \mathrm{~atm}$ (NPT ensemble) for $5 \mathrm{~ns}$ fixing the position of Fmoc-SOMe. A 2 fs time step and periodic boundary conditions in the three spatial coordinates ${ }^{61}$ were used as well as a $12 \AA$ cut-off for non-bonded interactions. Langevin dynamics were used for the temperature control and Langevin piston Nose-Hoover algorithm was used to keep the pressure constant. ${ }^{62}$ The density of both systems was calculated after this equilibration step to be $0.943 \mathrm{~g} / \mathrm{ml}$ for water and $0.805 \mathrm{~g} / \mathrm{ml}$ for the octanol, which are reasonably similar to the experimental values of $1.000 \mathrm{~g} / \mathrm{ml}$ and $0.824 \mathrm{~g} / \mathrm{ml}$, respectively.

Although water is known to be soluble in octanol $(0.255$ mole fraction) and hence, experimentally, some water will pass to the octanol phase, it has been demonstrated that computationally the addition of the appropriate number of water molecules into the octanol phase has a minimum effect, and the results for the dry octanol are slightly more accurate than those with the wet octanol. ${ }^{63}$

The FEP calculations were carried out using the same general MD parameters described before. Alchemical transformation was applied to decouple the Fmoc-S-OMe from the solvent. A decoupling constant $d \lambda=0.0625$ was used, giving

a)

Figure 6. Fmoc-S-OMe (Fmoc in red and Ser in blue) systems in (a) water and (b) octanol. Hydrogens are removed in the solvents for clarity. 
rise to up to 16 windows in the disappearance of Fmoc-S-OMe (from $\lambda=0$, Fmoc-S-OMe in solution, to $\lambda=1$, no Fmoc-S-OMe in solution) and a further 16 windows for the appearance of Fmoc-S-OMe, i.e., reverse FEP. Each window was equilibrated for 250,000 steps (0.5 ns) and simulated for a total of $4,250,000$ (8.5 ns). A soft-core potential ${ }^{64,65}$ was applied to avoid end-point problems ${ }^{66}(\lambda=0.5-1)$ and simple overlap sampling (SOS) ${ }^{67}$ was used to combine both, forwards and reverse simulations. The ParseFEP plugin version 1.9 was used for the error calculation. ${ }^{68}$

The logarithm of the partition coefficients $(\log P)$ is used for the comparison between the experimental and the MD-FEP determination (see ESI for details of the experimental determination and calculation of the values). Experimentally, the Log P for Fmoc-S-OMe was determined as $-1.4 \pm 0.1$, which compares favourably to the value calculated via MD-FEP $(-0.8$ \pm 0.2 ). The error between the experimental and calculated values ranges from 0.3 to 0.7 , which is of a similar magnitude to that obtained for a series of small alkanes reported in the literature. ${ }^{63}$ Given the much larger size of the Fmoc-S-OMe molecule (relative to the alkane series) and the amphiphilic nature of the molecules, the level of agreement between the experimental and calculated values is excellent.

\section{Validation via Self-Assembly Simulations.}

As described in the introduction, the parameterization of the Fmoc moiety was carried out in order to be able to implement MD simulations of Fmoc-peptides to allow greater insights into the self-assembling process of these molecules as well as of the final structures formed. Therefore, it is critical to validate the Fmoc parameters in a simulation of selfassembling Fmoc containing molecules.

Fmoc-S-OH and Fmoc- $\mathrm{Y}-\mathrm{OH}$ only contain one amino acid, serine (S), and tyrosine $(\mathrm{Y})$, respectively. The former one selfassembles into spherical aggregates in solution, ${ }^{4}$ while the latter, into fibres. ${ }^{27}$ They were chosen due to their simplicity (only one amino acid) and the simplicity of the structures formed, spheres and fibres, which can be easily compared with experimental data. The computational results will be compared with the experimental results published by AbulHaija et al. on the aggregation of Fmoc-S-OH, specifically, the AFM image presented in this publication (Figure ESI-4); ${ }^{4}$ and with the experimental results published by Yang et al. on the self-assembly of Fmoc-Y-OH into fibres. ${ }^{27}$

MD self-assembly simulations. Both self-assembling simulation was constructed with 120 randomly distributed Fmoc-S-OH or Fmoc-Y-OH molecules and solvated using $\mathrm{VMD}^{60}$ (Figure 7b, $0 \mathrm{~ns}$ ) with TIP3P water. ${ }^{48}$ Following this, the systems were minimized with the steepest descent technique to avoid bad contacts in the starting structure and then gradually ( $5 \mathrm{~K}$ every $1 \mathrm{ps}$ ) heated from 0 to $298 \mathrm{~K}$ over $60 \mathrm{ps}$ at $1 \mathrm{~atm}$. Finally, the systems were simulated for $300 \mathrm{~ns}$ in the NPT ensemble ( $1 \mathrm{~atm}, 298 \mathrm{~K}$ ). All other MD parameters were the same as those described in the partition coefficient section. Each system, after the heating phase, has a size of $~$ $83 \times 84 \times 83 \AA$ and, hence, the concentration of Fmoc-S-OH and $\mathrm{Fmoc}-\mathrm{Y}-\mathrm{OH}$ is $0.34 \mathrm{M}$. The $300 \mathrm{~ns}$ simulations were a)

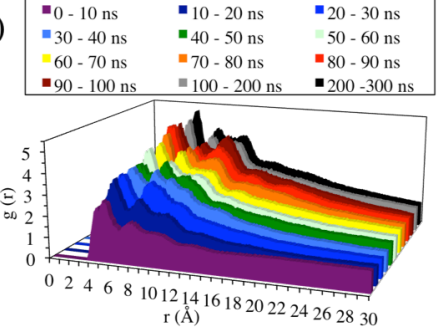

b)

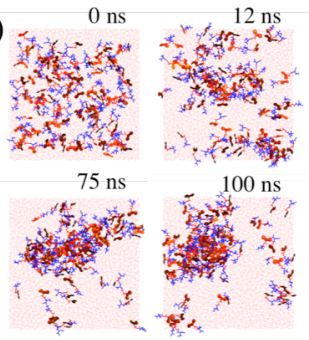

c)
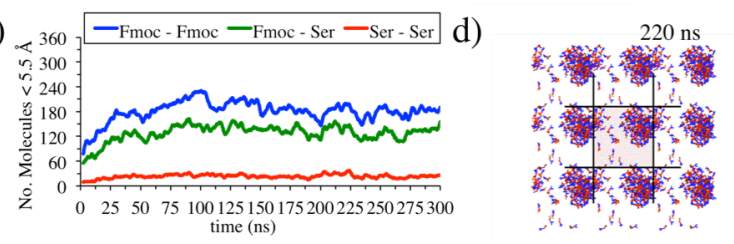

Figure 7. Results of the Fmoc-S-OH simulation: RDF analysis (a), proximity analysis (c) and snapshots of the simulation (b and d, Fmoc in red and S-OH in blue). The last snapshot d) shows also the periodic images in the $x y$-plane for clarity. Bigger sized snapshots are included in Fig. ESI-5.

analysed using the radial distribution function (RDF) using the $\mathrm{C}_{12}$ (see Figure 1 for atom names) of the aromatic group in the Fmoc moiety to measure the aggregation and proximity of the aromatic groups at different stages of the simulation. A proximity analysis (Figure 7c) is also presented, which accounts for the number of groups within $5.5 \AA$ through the simulation. In the case, of Fmoc - Fmoc proximity, a distance of $5.5 \AA$ between the centres of two aromatic groups is considered as a limiting distance for possible presence of $\pi$-stacking interactions.

Fmoc-S-OH simulation results and comparison with experiment. The snapshots of the simulation show how after 220 ns the Fmoc-S-OH form a spherical aggregate (Figure 7d), which is consistent with the experimental results.

The radial distribution functions for the first $100 \mathrm{~ns}$ are calculated by taking snapshots of the systems every $0.01 \mathrm{~ns}$ (i.e., 1000 frames per $10 \mathrm{~ns}$ ), whereas for the distributions from $100-300$ ns are based on snapshots of the system taken every 0.1 ns (i.e. 1000 frames per 100 ns). The lower sampling frequency in the latter stages of the simulation was due to the relative stability of the system after $100 \mathrm{~ns}$.

The RDF analysis (Figure 7a) shows three distinctly different sizes of aggregates through the simulation: peak 1 $(3.5-7 \AA)$, peak $2(7-9 \AA)$ and peak $3(9-12 \AA)$. Peak 1 , corresponds to Fmoc groups in direct contact. This peak increases rapidly at the beginning of the simulation $(20-50$ ns) and represents an early stage of the process where molecules form small aggregates. This initial peak reaches a plateau while peak 2 and peak 3 increase due to the junction of the small aggregates (e.g., Figure 7b, 12 ns). After $\sim 50 \mathrm{~ns}$, these latter peaks also begin to plateau and peak 1 starts to increase further, which corresponds to the formation of a single large aggregate (Figure 7b, $75 \mathrm{~ns}$ ). After $\sim 80 \mathrm{~ns}$ the variations in the radial distribution function are minimal and the overall shape and magnitude of the distribution remains consistent through the $100-200$ ns and $200-300$ ns plots, suggesting that the system is equilibrated following the 
formation of a spherical aggregate (Figure 7b, 100ns). A fourth and a fifth peak around $12-16 \AA$ and $16-20 \AA$, respectively, is also observed in the equilibrated parts of the graph.

The higher peak 3 between 80 and 100 ns in comparison with the $100-300$ ns results could be due to an elongation of the aggregated spheres to an ellipse like aggregate. However, as the simulation continues and is averaged over longer timescales, this elongation effect is no longer present.

In the proximity analysis (Figure 7c), due to the different size of the groups analysed, it is difficult to compare the relative importance of the role of the contacts studied. However, the Ser - Ser contacts are mostly negligible through the whole simulation with a maximum value 35 , which is equivalent to 0.3 interactions/molecule, while the Fmoc Fmoc and Fmoc - Ser reach maxima of $\sim 1.9$ and $\sim 1.3$ respectively. This suggests that the Ser groups tend to be exposed to the solvent, forming H-bonds, instead of interacting with other Ser groups.

The proximity analysis (Figure 7c) shows a rapid increase in the Fmoc - Fmoc proximity as well as the Fmoc - Ser proximity, which is likely due to the arrangement achieved through the Fmoc $\pi$-stacking interactions. The proximity analysis reveals how the molecules aggregate quickly in the first 25 ns to relatively stable structures, until $\sim 50 \mathrm{~ns}$, where the aggregation is seen to increase further. This is in good agreement with the RDF analysis, which shows an early aggregation step at the same time of the simulation. Once the small aggregates are formed they coalesce to form larger aggregates (50 - 100 ns). Therefore, the Fmoc - Fmoc interactions remain constant while peak 2 of the RDF increases (Figure 7a). This suggests that this increase is not due to Fmoc interactions, which are mostly buried in the small aggregates, but once these small aggregates join, the Fmoc groups of the aggregates interact inside the larger aggregate (as peak 1 in the RDF at 50 to $100 \mathrm{~ns}$ ). The highest level of Fmoc - Fmoc aggregation is reached around 100 ns. This high number of interactions slightly decreases, in a process of equilibrating the aggregate, and remains relatively unchanged, apart from normal fluctuations, at $\sim 180 \mathrm{Fmoc}-$ Fmoc interactions ( $\sim 1.5$ interactions/molecule) from 110 - $300 \mathrm{~ns}$ forming and aggregate around $40 \AA$.

Although specific interactions are not determined in detail, the proximity analysis can be used to identify the orientations of the Fmoc - Fmoc systems (Figure 4) that most closely correlate with the simulations results. All of the orientations are expected to have a high number of Fmoc - Fmoc interactions and hence, the other two parameters provide more insight, using the former as a reference. The Ser - Ser interactions are negligible and hence, parallel stacking orientations can be excluded. In contrast, the number of Fmoc - Ser interactions is similar to that of the Fmoc - Fmoc interactions, which suggest that $\mathrm{T}$ forms are also not dominant in the simulation. Therefore, a dominant non-T antiparallel orientation most readily explains the proximity analysis results.

The AFM image of Fmoc-S-OH published by Abul-Haija et al. ${ }^{4}$ shows many small aggregates and only a few larger ones, but not aggregates of intermediate size. While care should be taken with the interpretation of AFM images because of possible drying effects influencing the size distribution, the mechanism observed in the simulation reveals how these larger aggregates are formed when many small aggregates coalesce, which is consistent with this experimental observation. Furthermore, the simulation is also in good agreement with the shape of the aggregates, which are mostly spherical, with a limited number showing a degree of ellipticity. However, in addition to this data, the simulation of the Fmoc-S-OH system also provides insight into which interactions are driving the different stages of the process. That is, the initial formation of the small aggregates is driven by the $\pi$-stacking interactions of the Fmoc moieties, followed by the $\mathrm{H}$-bonding interactions between the Ser-OH moieties which allow the small aggregates to come together to finally be stabilized through additional $\pi$-stacking between the Fmoc moieties. Therefore, in addition to the simulation providing good agreement with the experimental results it also is able to contribute unique insights into the molecular level information, which is otherwise inaccessible experimentally.

Fmoc-Y-OH simulation results and comparison with experiment. This simulation requires up to $300 \mathrm{~ns}$ to reach an equilibrated structure (Figure $8 d$ ). For this simulation, the RDFs are calculated every $0.01 \mathrm{~ns}$ for the first $50 \mathrm{~ns}$ (i.e., 1000 frames per $10 \mathrm{~ns}$ ) and every 0.05 for the rest of the simulation (i.e., 1000 frames per $50 \mathrm{~ns}$ ).

The RDF analysis of Fmoc-Y-OH (Figure 8a) is similar to the Fmoc-S-OH (Figure 7a) for the first $10 \mathrm{~ns}$, showing three peaks of similar intensity, where peak $1(3.5-7 \AA)$ is dominant due to the first stages of aggregation between $\mathrm{Fmoc}-\mathrm{Y}-\mathrm{OH}$ molecules. After the first 10 ns of the simulation, peak 3 ( $9-$ $12 \AA)$ increases faster than peak 2 (7-9 $\mathrm{A})$, in contrast to Fmoc-S-OH, due to the elongated nature of the aggregates forming (Figure 8b, 25 ns). Between 40 ns and 150 ns both peaks are of similar intensity, while peak 1 clearly decreases, corresponding to the formation of a fibre like structure from the aggregates (Figure $8 \mathrm{~b}, 75 \mathrm{~ns}$ and $150 \mathrm{~ns}$ ). At the end of the
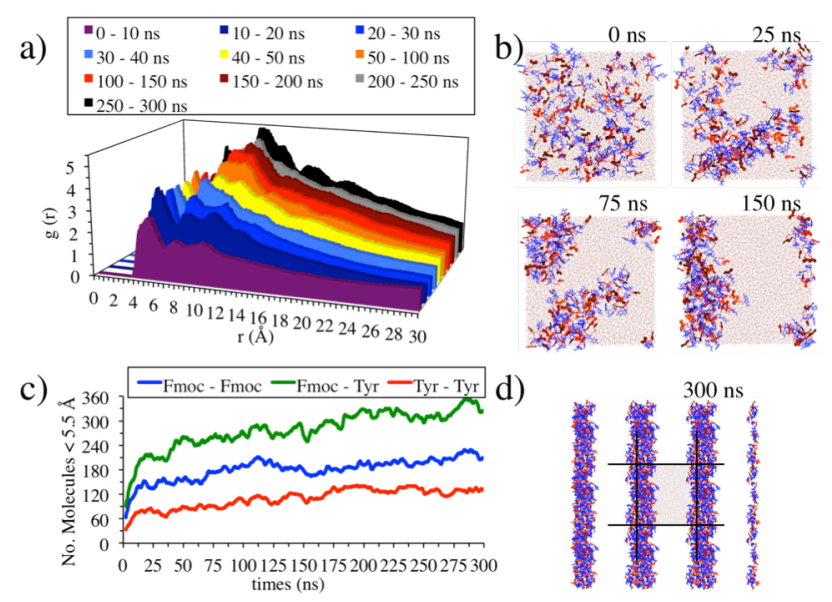

Figure 8. Results of the Fmoc-Y-OH simulation: RDF analysis (a), proximity analysis (c) and snapshots of the simulation ( $b$ and d, Fmoc in red and $\mathrm{Y}-\mathrm{OH}$ in blue). The last snapshot d) shows also the periodic images in the $x y$-plane for clarity. Bigger sized snapshots are included in Fig. ESI-6. 
simulation peak 1 increases again but remains below peak 3 and peak 2, which is the highest peak (Figure 8a, grey and black). In the final $50 \mathrm{~ns}$ two additional peaks form around $12-$ $16 \AA$ and $16-20 \AA$. These peaks were also present in the Fmoc-S-OH simulations but they are clearly more prominent in the last $50 \mathrm{~ns}$ of the $\mathrm{Fmoc}-\mathrm{Y}-\mathrm{OH}$ simulation. This is consistent with the formation of a more ordered, equilibrated fibre observed at the end of the simulation (Figure 8d).

The Fmoc-Y-OH proximity analysis (Figure $8 \mathrm{c}$ ) shows a rapid increase in the three proximity curves during the first $10 \mathrm{~ns}$ of the simulation. This can be attributed to the fast aggregation of the aromatic groups of both, the Fmoc and the Tyr. This increment at the start is faster than in the case of Fmoc-S-OH. In addition, the amino acid - amino acid proximity (Tyr - Tyr), unlike in the case of the Ser - Ser, is of importance, reaching an averaged 120 proximity counts over the last 100 ns of the simulation. These two effects are caused by the hydrophobicity of the tyrosine side chain and its ability to form $\pi$-stacking interactions. Despite the fact that the initial aggregation occurs more rapidly, relative to $\mathrm{Fmoc}-\mathrm{S}-\mathrm{OH}$, the proximity curves continue to increase throughout the simulation, which shows that the more complex fibre structure requires more time to equilibrate than the simple spherical aggregate of $\mathrm{Fmoc}-\mathrm{S}-\mathrm{OH}$. The $\mathrm{Fmoc}-\mathrm{Fmoc}$ proximity values are similar to those for the Fmoc-S-OH ( 180), but the interactions that involve the Tyr are much more significant than the analogous interactions involving Ser: Fmoc - Ser reaches $\sim 120$, while Fmoc - Tyr, $\sim 360$; Ser - Ser interactions equilibrate at $\sim 25$ whereas Tyr $-\mathrm{Tyr}$ are $\sim 120$. This clearly shows the importance of the aromatic side chain in forming fibres rather than spherical aggregates.

The important role of the three types of interactions identified with the proximity analysis (Figure $8 \mathrm{c}$ ) makes it difficult to relate the results with the Fmoc - Fmoc orientations studied before (Figure 4), as was done for the Fmoc-S-OH system. This is because the arrangement of simple dimers cannot explain the abundance of the three types of interactions as these probably arises from interactions between multiple molecules (e.g., twisted-stacks).

The ability to reproduce two different experimentally known nanostructures from closely related systems clearly demonstrates that the parameterization has achieved the correct balance and description of the important interactions to correctly describe the role of Fmoc in forming these different nanostructures.

\section{Conclusions}

A new protocol for the parameterization of amphiphilic molecules to be used in self-assembling studies has been developed. A set of parameters for the Fmoc moiety has been derived for the CHARMM force field using this protocol. The parameters are shown to be able to reproduce intermolecular interactions by reproducing QM binding energies between the moiety and water and between two Fmoc moieties, and to reproduce the flexibility of the Fmoc group by comparing dihedral distributions in $\mathrm{MD}$ simulations and their $\mathrm{MM}$ energetic profile with the corresponding QM dihedral scans. Furthermore, the Fmoc parameterization presented in this paper has successfully reproduced thermodynamic parameters directly related to the self-assembling behaviour and experimental results involving self-assembling of the moiety linked to an amino acid. This includes achieving different nanostructures for different systems, spheres for Fmoc-S-OH and fibres for Fmoc- $\mathrm{Y}-\mathrm{OH}$, consistent with the experimental findings for these systems. The validity of the parameters supports the modifications made in the protocol for this type of molecule and suggest a new procedure for the future parameterization of other amphiphilic moieties.

\section{Acknowledgements}

The authors gratefully acknowledge the financial support by the EC 7th Framework Programme Marie Curie Actions via the European ITN SMARTNET No. 316656 and EMERgE/ERC No. 258775. Results were obtained using the EPSRC funded ARCHIE-WeSt High Performance Computer (www.archiewest.ac.uk). EPSRC grant no. EP/K000586/1.

\section{References}

1. M. Zelzer and R. V. Ulijn, Chem. Soc. Rev., 2010, 39, 33513357.

2. G. M. Whitesides and B. Grzybowski, Science, 2002, 295 , 2418-2421.

3. J. E. Gough, A. Saiani and A. F. Miller, Bioinspired, Biomimetic and Nanobiomaterials, 2011, 1, 4-12.

4. Y. M. Abul-Haija, S. Roy, P. W. Frederix, N. Javid, V. Jayawarna and R. V. Ulijn, Small, 2014, 10, 973-979.

5. M. Hughes, L. S. Birchall, K. Zuberi, L. A. Aitken, S. Debnath, N. Javid and R. V. Ulijn, Soft Matter, 2012, 8, 11565-11574.

6. M. Reches and E. Gazit, Science, 2003, 300, 625-627.

7. M. Hughes, P. W. J. M. Frederix, J. Raeburn, L. S. Birchall, J. Sadownik, F. C. Coomer, I. H. Lin, E. J. Cussen, N. T. Hunt, T. Tuttle, S. J. Webb, D. J. Adams and R. V. Ulijn, Soft Matter, 2012, 8, 5595-5602.

8. Y. R. Zhao, J. Q. Wang, L. Deng, P. Zhou, S. J. Wang, Y. T. Wang, H. Xu and J. R. Lu, Langmuir, 2013, 29, 1345713464.

9. M. McCullagh, T. Prytkova, S. Tonzani, N. D. Winter and G. C. Schatz, J. Phys. Chem. B, 2008, 112, 10388-10398.

10. Y. S. Velichko, S. I. Stupp and M. O. de la Cruz, J. Phys. Chem. B, 2008, 112, 2326-2334.

11. O.-S. Lee, V. Cho and G. C. Schatz, Nano Lett., 2012, 12 , 4907-4913.

12. T. Yu and G. C. Schatz, J. Phys. Chem. B, 2013, 117, 1405914064.

13. P. W. J. M. Frederix, R. V. Ulijn, N. T. Hunt and T. Tuttle, J. Phys. Chem. Lett., 2011, 2, 2380-2384.

14. P. Frederix, W. J. M., G. G. Scott, Y. M. Abul-Haija, D. Kalafatovic, C. G. Pappas, N. Javid, N. T. Hunt, R. V. Ulijn and T. Tuttle, Nature Chem., 2015, 7, 30-37.

15. D. E. Lopez-Perez, G. Revilla-Lopez, I. W. Hamley and C. Aleman, Soft Matter, 2013, 9, 11021-11032. 
16. O.-S. Lee, Y. Liu and G. C. Schatz, J. Nanopart. Res., 2012, 14, 1-7.

17. O.-S. Lee, S. I. Stupp and G. C. Schatz, J. Am. Chem. Soc., 2011, 133, 3677-3683.

18. T. Yu and G. C. Schatz, J. Phys. Chem. B, 2013, 117, 90049013.

19. T. Yu, O. S. Lee and G. C. Schatz, J. Phys. Chem. A, 2013, 117, 7453-7460.

20. R. Orbach, L. Adler-Abramovich, S. Zigerson, I. MironiHarpaz, D. Seliktar and E. Gazit, Biomacromolecules, 2009, 10, 2646-2651.

$21 . \quad J$. D. Hartgerink, E. Benlash and S. L. Stupp, Science, 2001, 294, 1684-1688.

22. F. Versluis, H. R. Marsden and A. Kros, Chem. Soc. Rev., 2010, 39, 3434.

23. R. V. Ulijn and A. M. Smith, Chem. Soc. Rev., 2008, 37, 664-675.

24. C. Tang, R. V. Ulijn and A. Saiani, Langmuir, 2011, 27, 14438-14449.

25.

C. Tang, R. V. Ulijn and A. Saiani, Eur. Phys. J. E, 2013, 36, 1-11.

26. M. Caruso, E. Gatto, E. Placidi, G. Ballano, F. Formaggio, C. Toniolo, D. Zanuy, C. Alemán and M. Venanzi, Soft Matter, 2014, 10, 2508-2519.

27. Z. Yang, H. Gu, D. Fu, P. Gao, J. K. Lam and B. Xu, Adv. Mater, 2004, 16, 1440-1444.

28. S. Sutton, N. L. Campbell, A. I. Cooper, M. Kirkland, W. J. Frith and D. J. Adams, Langmuir, 2009, 25, 10285-10291.

29. S. Fleming and R. V. Ulijn, Chem. Soc. Rev., 2014, 43, 81508177.

30. S. Fleming, S. Debnath, P. W. J. M. Frederix, T. Tuttle and R. V. Ulijn, Chem. Commun., 2013, 49, 10587-10589.

31. X. J. Mu, K. M. Eckes, M. M. Nguyen, L. J. Suggs and P. Y. Ren, Biomacromolecules, 2012, 13, 3562-3571. H. X. Xu, A. K. Das, M. Horie, M. Shaik, A. M. Smith, Y. Luo, X. Lu, R. Collins, S. Y. Liem, A. Song, P. L. A. Popelier, M. L. Turner, P. Xiao, I. A. Kinloch and R. V. Ulijn, Nanoscale, 2010, 2, 960-966.

33. V. Castelletto, C. Moulton, G. Cheng, I. Hamley, M. R. Hicks, A. Rodger, D. E. López-Pérez, G. Revilla-López and C. Alemán, Soft Matter, 2011, 7, 11405-11415.

34. A. Mackerell, D. Bashford, M. Bellot, R. Dunbrack, J. Evanseck, M. Field, J. Gao, H. Guo, S. Ha, D. JosephMcCarthy, L. Kuchnir, K. Kuczera, F. T. K. Lau, C. Mattos, S. Michnick, T. Ngo, D. T. Nguyen, B. Prodhom, W. E. E. Reiher, B. Roux, M. Schlenkrich, J. C. Smith, R. Stote, J. Straub, M. Watanabe, J. Wio' rkiewicz-Kuczera, D. Yin and M. Karplus, J. Phys. Chem. B, 1998, 102, 3586.

35. A. R. Leach, Molecular modelling: principles and applications, Prentice Hall, Harlow [etc.], 2001.

36. S. A. Adcock and J. A. McCammon, Chem. Rev., 2006, 106, 1589-1615.

W. F. van Gunsteren, D. Bakowies, R. Baron, I. Chandrasekhar, M. Christen, X. Daura, P. Gee, D. P. Geerke, A. Glättli and P. H. Hünenberger, Angew. Chem., 2006, 45, 4064-4092.

38 B. R. Brooks, C. L. B. III, A. D. M. Jr, L. Nilsson, R. J. Petrella, B. Roux, Y. Won, G. Archontis, C. Bartels, S. Boresch, A. Caflisch, L. Caves, Q. Cui, A. R. Dinner, M. Feig, S. Fischer, J. Gao, M. Hodoscek, W. Im, K. Kuczera, T. Lazaridis, J. Ma, V. Ovchinnikov, E. Paci, R. W. Pastor, C. B. Post, J. Z. Pu, M. Schaefer, B. Tidor, R. M. Venable, H. L. Woodcock, X.

Wu, W. Yang, D. M. York and M. Karplus, J. Comput. Chem., 2009, 30, 1545-1614.

39.

40. 2000, 56, 257-265

K. Vanommeslaeghe, E. Hatcher, C. Acharya, S. Kundu, S. Zhong, J. Shim, E. Darian, O. Guvench, P. Lopes, I. Vorobyov and A. D. Mackerell, J. Comput. Chem., 2010, 31, 671-690.

41. E. T. Prates, P. C. Souza, M. Pickholz and M. S. Skaf, Int. J. Quantum Chem., 2011, 111, 1339-1345.

42. A. Hansson, P. C. Souza, R. L. Silveira, L. Martinez and M. S. Skaf, Int. J. Quantum Chem., 2011, 111, 1346-1354.

43. S. Grimme, J. Comput. Chem., 2006, 27, 1787-1799.

44. A. Schäfer, H. Horn and R. Ahlrichs, J. Chem. Phys., 1992, 97, 2571-2577.

TURBOMOLE, 6.3.1 (2012), TURBOMOLE GmbH.

46. R. Ahlrichs, M. Bär, M. Häser, H. Horn and C. Kölmel, Chem. Phys. Lett., 1989, 162, 165-169.

47. J. C. Phillips, R. Braun, W. Wang, J. Gumbart, E. Tajkhorshid, E. Villa, C. Chipot, R. D. Skeel, L. Kalé and K. Schulten, J. Comput. Chem., 2005, 26, 1781-1802.

48. W. L. Jorgensen, J. Chandrasekhar, J. D. Madura, R. W. Impey and M. L. Klein, J. Chem. Phys., 1983, 79, 926-935.

49. M. D. Hanwell, D. E. Curtis, D. C. Lonie, T. Vandermeersch, E. Zurek and G. R. Hutchison, J. Cheminform., 2012, 4, 17. Gaussian 09, (2009), Gaussian, Inc.

50.

51.

52.

A. D. Becke, The Journal of Chemical Physics, 1993, 98, 5648-5652.

C. Lee, W. Yang and R. G. Parr, Physical Review B, 1988, 37, 785.

53. W. J. Hehre, R. Ditchfield and J. A. Pople, The Journal of Chemical Physics, 1972, 56, 2257-2261.

P. C. Hariharan and J. A. Pople, Theor. Chim. Acta, 1973, 28, 213-222.

J. Sangster, J. Phys. Chem. Ref. Data, 1989, 18, 1111-1229. K. B. Lodge, J. Chem. Eng. Data, 1999, 44, 1321-1324.

D. J. Edelbach and K. B. Lodge, Phys. Chem. Chem. Phys., 2000, 2, 1763-1771.

R. W. Zwanzig, J. Chem. Phys., 1954, 22, 1420-1426.

R. W. Zwanzig, J. Chem. Phys., 1955, 23, 1915-1922.

60. W. Humphrey, A. Dalke and K. Schulten, J. Mol. Graphics, 1996, 14, 33-38.

61. O. N. de Souza and R. L. Ornstein, Biophys. J., 1997, 72, 2395-2397.

62. S. E. Feller, Y. Zhang, R. W. Pastor and B. R. Brooks, J. Chem. Phys., 1995, 103, 4613-4621.

63. N. Bhatnagar, G. Kamath, I. Chelst and J. J. Potoff, J. Chem. Phys., 2012, 137.

64. T. C. Beutler, A. E. Mark, R. C. van Schaik, P. R. Gerber and W. F. van Gunsteren, Chem. Phys. Lett., 1994, 222, 529539.

65. J. W. Pitera and W. F. van Gunsteren, Mol. Simul., 2002, 28, 45-65.

66. C. Chipot and A. Pohorille, Free energy calculations, Springer, 2007.

67. C. Lee and H. Scott, J. Chem. Phys., 1980, 73, 4591-4596.

68. P. Liu, F. o. Dehez, W. Cai and C. Chipot, J. Chem. Theory Comput., 2012, 8, 2606-2616. 